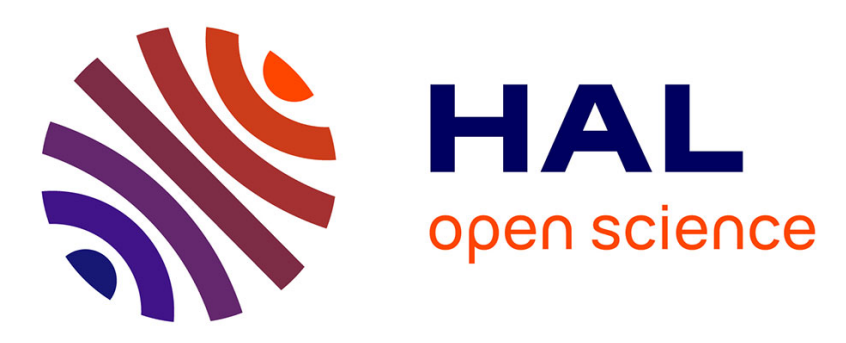

\title{
Espaces documentaires, espaces de savoir, espaces d'expérience: vers une (re)définition du modèle des CDI?
}

\author{
Yolande Maury
}

\section{- To cite this version:}

Yolande Maury. Espaces documentaires, espaces de savoir, espaces d'expérience: vers une (re)définition du modèle des CDI?. Actes du 39ème congrès CAIS-ACSI: Les intersections, gens, lieux, informations, Jun 2011, Fredericton, Nouveau-Brunswick, Canada. 10.29173/cais581 . hal00986160

\section{HAL Id: hal-00986160 https://hal.science/hal-00986160}

Submitted on 1 May 2014

HAL is a multi-disciplinary open access archive for the deposit and dissemination of scientific research documents, whether they are published or not. The documents may come from teaching and research institutions in France or abroad, or from public or private research centers.
L'archive ouverte pluridisciplinaire HAL, est destinée au dépôt et à la diffusion de documents scientifiques de niveau recherche, publiés ou non, émanant des établissements d'enseignement et de recherche français ou étrangers, des laboratoires publics ou privés. 


\title{
Espaces documentaires, espaces de savoir, espaces d'expérience : vers une (re)définition du modèle des CDI?
}

\begin{abstract}
Résumé : Cette communication interroge les Centres de Documentation et d'Information (CDI) en tant qu'espaces physiques et symboliques, au cœur de l'organisation et la construction des savoirs. Ceci, en appui sur deux recherches consécutives (2001-2005; 2006-2010) dans le cadre de l'enseignement secondaire français. Des évolutions sont perceptibles qui questionnent les modèles établis.

Abstract: This presentation explores the Centres de Documentation et d'Information (CDI) as physical and symbolic spaces, at the heart of knowledge organization and construction. This research is based on two previous and consecutive research projects $(2001-2005 ; 2006-2010)$ on high school education in France. Findings show evolutions that question established models.
\end{abstract}

Alors que la réflexion actuelle autour des learning centers et des bibliothèques «troisième lieu » interroge les modèles classiques de bibliothèques, le lieu CDI se trouve aujourd'hui au centre de bien des attentions. Présenté successivement comme un « service », un « centre », plus récemment un «cœur» ou même le "poumon » de l'établissement, son rôle central ne semble plus être remis en cause alors que sa mutation annoncée en E-CDI virtuel, rendant la documentation présente partout et remettant en cause l'idée de centre, pouvait laisser envisager à terme sa disparition (Navarro 2009).

En appui sur les résultats de deux recherches (2001-2005 et 2006-2010), nous proposons une réflexion sur le territoire de l'information-documentation scolaire, avec l'objectif de mettre en évidence ce qu'une réflexion sur l'espace CDI peut apporter à la compréhension de l'organisation et de la construction des savoirs, orientés vers l'acquisition d'une culture informationnelle. Alors que notre culture tend à minimiser l'importance de l'espace, le reléguant souvent dans l'informel (Hall 1984), il s'agit de lui donner une visibilité en tant que dimension cachée de la culture [informationnelle]. Suivant l'idée que l'espace ne fait pas seulement communiquer, au sens élémentaire du terme, mais qu'il est révélateur des intentions des acteurs et organise les pratiques et les institutions.

Nous poserons d'abord quelques éléments théoriques permettant d'éclairer le concept d'espace, documentaire notamment, en lien avec celui de culture informationnelle. Après avoir présenté le contexte de la recherche et les méthodologies retenues, nous dégagerons, d'une recherche à l'autre, les dynamiques qui se créent autour des espaces documentaires, considérés dans leurs dimensions, physique et symbolique, et appréhendés en tant qu'éléments structurants des pratiques, significatifs d'une culture informationnelle en action. Ce qui devrait nous amener à interroger le modèle de bibliothèque scolaire sous-jacent.

\section{Contextes, repères théoriques}


« Ce qui est visible, pensable, et entendu nous parle [...] toujours d'une façon directe d'un arrière-fond qui supporte la vision, la pensée, l'entendement » (Jager 1998). Dans le cas des espaces documentaires, il s'agit à partir du visible, du pensable, d'accéder au sens de ces espaces et pour cela traduire le visible en lisible. Car même si son importance est minimisée, l'espace transmet des messages (Hall 1971). Il le fait suivant une sémantique tantôt explicite, tantôt implicite, l'acquisition d'une culture informationnelle contribuant à désimpliciter ce qui est transparent et/ou implicite.

Par culture informationnelle, nous entendons l'ensemble des connaissances à partager pour être à l'aise, vivre, évoluer dans un monde où l'information occupe une place centrale. Ce qui représente bien plus que la littératie informationnelle et que la culture de l'information. Débordant de la recherche d'information et de la culture de la recherche, la culture informationnelle implique une dimension sociale et culturelle (Baltz 1998, Juanals 2003). Référée à des modèles sociaux partagés de comportements, de normes, de valeurs, elle est introduction à un monde (au sens de «devenir membre ») : l'informationnel faisant non seulement «signe» et informant l'esprit, mais modifiant la perception du monde et participant d'un processus de transformation (empowerment) (Maury 2010)

Métaphores du savoir, les espaces documentaires constituent une entrée dans la culture. Territoires symboliques, organisés selon des modèles et des règles, rigoureusement structurés, ils participent au "pouvoir communiquer» du documentaliste. Espaces d'énonciation, avec leurs codes et leurs règles, décomposables et analysables, ils sont comme la culture, déchiffrables (Hall 1971): leur disposition, les objets documentaires et activités qui les animent sont autant de traces descriptibles qui permettent de remonter, des objets et des pratiques, à la culture. Espaces matériels et symboliques, ils sont aussi des espaces sociaux, lieux de contacts et d'expériences. Considérés globalement enfin en référence à la différenciation espace public/espace privé (Goffman 1973), ils semblent fonctionner comme des espaces mixtes. Et si pour les caractériser les dénominations varient («non lieu » (Fabre 2006), go between...), et qu'une volonté existe d'en faire des learning centers, leurs métamorphoses semblent interroger le modèle classique de la bibliothèque scolaire, à l'heure de la bibliothèque « troisième lieu » (Putnam 2003, Servet 2010).

\section{Méthodologie, résultats, perspectives}

Les résultats présentés s'appuient sur deux recherches ayant pour objet les pratiques d'éducation à l'information dans l'enseignement secondaire français : un travail universitaire réalisé pour l'obtention du doctorat, avec l'objectif de faire émerger les dynamiques des pratiques en étudiant les interactions environnement/enseignant/élèves ; une recherche dans le cadre de l'ERTé Culture informationnelle et curriculum documentaire (Lille 3, Béguin dir.), opérant un recentrage sur la culture informationnelle en action, avec une approche anthropologique, attentive aux dimensions proxémique et temporelle.

Notre corpus est donc double, constitué d'une série d'observations réalisées de manière ethnographique par immersion dans le quotidien des CDI, croisées avec des entretiens pour asseoir la validité des données. Pour chaque recherche, quatre enseignantes-documentalistes de l'académie de Paris ( $1^{\text {ère }}$ recherche, toutes chevronnées ; deux confirmées et deux débutantes, ensuite) ont été observées sur la durée. Les entretiens structurés, en fin de parcours, donnent leur regard sur les pratiques (définition culture informationnelle, retour sur les séances). Des écrits réflexifs et extraits de conversations informelles complètent les 
données. Pour l'analyse, nous avons procédé à une lecture en positif, attentive à repérer des dynamiques plutôt que des manques, ce qui permet de rendre compte des pratiques entre permanence et changement et de repérer des émergences.

\subsection{Contextes proxémiques, sémiotique de l'espace, ancrage spatial des savoirs}

Espace semi-public, le CDI est un espace de circulation, lieu de rencontres avec les documents, l'information et les autres. L'idée de territorialité, variable d'un CDI à l'autre, est très forte.

L'existence d'un «accent spatial», rendant compte de l'empreinte marquée du documentaliste, est confirmée au fil des recherches. Espace dont la géographie est liée aux déplacements, il offre par son organisation à la fois une mise en scène des savoirs et un balisage des parcours. Un premier constat met en évidence deux types de mise en espace (éclaté multifonctions; et fortement différencié opposant fonds physique et virtuel) à mettre en lien avec l'approche des parcours de formation. Le sens de l'évolution semble orienter vers une approche intégrée, le développement des ressources numériques permettant cette flexibilité. Un second constat porte sur la perte de sens intervenant en cas de délocalisation en salle de classe ou informatique. Ces délocalisations, nombreuses aujourd'hui, sont rarement perçues positivement par les élèves, habitués à un lieu plus ouvert aux pratiques non formelles, moins délimitant.

Espace d'énonciation et de médiation, l'espace documentaire est une composante forte du repérage de l'univers documentaire : «lieu pratiqué » (De Certeau 1990), il trouve sens et identité par les activités qui s'y déroulent. Une confirmation : le rapport à l'informationdocumentation est d'abord physique, d'autant plus que les usagers sont jeunes, la construction du sens s'effectuant du sensible vers l'intelligible. Les visites guidées gardent ainsi leur intérêt en tant que parcours «initiatique » rendant la déambulation moins erratique, plus questionnante de la complexité positive du lieu, plus capacitante. Au-delà de ce premier contact, les expériences informationnelles au cœur de la culture informationnelle opèrent comme une série de rencontres qui dessinent des figures de sens, signifiantes lorsque normes, codes, savoirs sont acquis (Fabbri 1968). Cette sémiotique de la rencontre met en œuvre une double signification, à la fois dans le domaine de la mémoire et d'un savoir commun partagé. Les classifications documentaires sont un exemple de la série de médiations en jeu qui à la fois font "signe" aux usagers et expriment la force de l'héritage. Avançant comme une construction sociale masquée, ces médiations s'affirment comme un enjeu de savoir questionnant à la fois l'idée de transparence et d'opacité de l'espace.

\subsection{Espaces de culture, d'autonomie, de responsabilisation, d'expérience... la symbolique de l'espace au cœur de la culture informationnelle}

Dans sa dimension symbolique, l'espace documentaire est l'objet de nombreuses projections de la part des acteurs, significatives des conceptions de la culture informationnelle.

La perception de l'espace est généralement dynamique, liée à l'action, à ce qui peut être accompli en appui sur cet espace (Hall 1971). A la fois espace de responsabilisation, tourné vers la formation du citoyen, et d'autonomie, autour de l'idée "d'un être humain en devenir », le CDI est vu comme un espace potentiel (Berten 1999), avec outils et ressources 
au service d'un projet personnel. Se situant dans «un esprit de rencontre », il est facteur de contact culturel, orienté vers "un commun qui va évoluer ", ouvert à l'altérité, et support de créativité " exacerbée par les rencontres" (ERTé lycée Photo). Matière d'actions et d'échanges (Piponnier 2002), il est censé, au-delà des besoins d'information, éveiller la curiosité (Metzger 2006), donner des possibilités de se cultiver " y compris par hasard, par rebond... [...] en dehors des disciplines » (lycée D).

Espace de culture et lieu de vie à la fois, il semble occuper une position intermédiaire. "Objet transitionnel » (Winnicot 1986), rappelant «un petit peu la maison » (collège C), il offre un environnement bienveillant, à ancrage physique fort, et « introduit » à un monde, développant un sentiment d'appartenance. A la rencontre pratiques non formelles-pratiques formelles, il fait le lien intérieur-extérieur "l'école [n'étant] pas fermée au monde ", et est support d'apprentissage afin "d'amener l'élève à une réflexion sur ses pratiques » (ERTé collège 2). Espace mixte, il apparaît ainsi à la fois région antérieure, lieu de «représentation » pour le documentaliste, et région postérieure, espace profane pour élèves et enseignants (Mollard 1996).

D'une recherche à l'autre enfin, les évolutions perceptibles interrogent le modèle des CDI : mettant en avant une "culture de la lenteur", avec des pauses face au "flot d'informations » et à la pression scolaire ; et d'autre part une "culture de l'expérience ", articulant expériences informationnelles au sens classique et expériences partagées, autour de moments culturels, apportant un plus, tant au niveau émotionnel (Giboreau, Body 2007) que cognitif, intellectuel. Elles renvoient l'image d'un CDI intégré, favorisant un ancrage physique et émotionnel, loin toutefois de l'idée d'une bibliothèque «troisième lieu » en milieu éducatif, même si la référence à une « économie de l'expérience » peut paraître fondée.

\section{Références bibliographiques}

Berten, André. 1999. Dispositif, médiation, créativité : petite généalogie. Hermès, 25: 33-47.

Baltz, Claude. 1998. Une culture pour la société de l'information ? Position théorique, définition, enjeux. Documentaliste-Sciences de l'information, vol. 35, 2: 75-82.

Béguin, Annette (dir.). 2010. Rapport final ERTé Culture informationnelle et curriculum documentaire, Lille 3.

Certeau, Michel de. 1990. L'invention du quotidien. 1. Arts de faire. Gallimard.

Fabre, Isabelle. 2006. L'espace documentaire comme espace de savoir : itinéraires singuliers et imaginaires littéraires. Thèse de Doctorat en Sciences de l'information et de la communication, Toulouse 2 Le Mirail. (Direction de recherche : Viviane Couzinet).

Fabbri, Paolo. 1968. Considérations sur la proxémique. Langages, vol 3, $10: 65-75$.

Giboreau, Agnès, Body, Laurence. 2007. Le marketing sensoriel. De la stratégie à la mise en euvre. Vuibert.

Goffman, Erving. 1973. La mise en scène de la vie quotidienne, tome 1. La présentation de soi. Paris : Ed. de Minuit. 
Hall, Edward T. 1971. La dimension cachée. Paris : Seuil.

Hall, Edward T. 1984. Le langage silencieux. Paris : Seuil.

Jager, Bernd. 1998. La demeure et l'espace habité comme point de départ d'une psychologie humaniste. Recherches qualitatives, $18: 7-16$.

Juanals, Brigitte. 2003. La culture de l'information : du livre au numérique. Paris : Lavoisier.

Liquète, Vincent, Maury, Yolande. 2007. Le travail autonome. Armand Colin, 2007.

Maury, Yolande. 2010. Définition(s) de la culture informationnelle. In : Béguin, Annette (dir.). Rapport final ERTé Culture informationnelle et curriculum documentaire, Lille 3, décembre : 127-133.

Metzger, Jean-Paul. 2006. L'information documentation. In : Olivesi, Stéphane (dir.).

Sciences de l'information et de la communication : objets, savoirs, discipline. P.U.G : 43-61

Mollard, Michèle. 1996. Les CDI à l'heure du management. Villeurbanne : ENSSIB ; Paris : FADBEN.

Navarro, Annick. 2009. Documentaliste dans l'Education nationale en France : un statut d'enseignant, des fonctions hybrides et une identité incertaine. Rede de Bibliotecas escolares 5

Piponnier, Anne. 2002. Codes et usages topographiques au CDI. Cahiers pédagogiques, $\mathrm{n}^{\circ}$ spécial. (Quoi de neuf à la doc ?).

Putnam, Robert Feldstein, Lewis Cohen, Donald. 2003. Better together. Restoring the American Community. Simon \& Schuster.

Servet, Mathilde. 2010. Les bibliothèques troisième lieu. $B B F, 4: 57-63$ 